\title{
X MARMONON
}

Title: Effects of controlled dehydration on sleep quality and quantity: A polysomnographic study in healthy young adults

Author(s): Aristotelous, Panagiotis, Aphamis, George, Sakkas, Giorgos K., Andreou, Eleni, Pantzaris, Marios, Kyprianou, Theodoros, Hadjigeorgiou, Georgios M., Manconi, Mauro and Giannaki, Christoforos D

Copyright, publisher and additional information:

This is the peer reviewed version of the following article: Aristotelous, Panagiotis, Aphamis, George, Aristotelous $P$, Aphamis $G$, Sakkas GK, et al. Effects of controlled dehydration on sleep quality and quantity: A polysomnographic study in healthy young adults. J Sleep Res. 2018;00:e12662, which has been published in final form at https://doi.org/10.1111/jsr.12662. This article may be used for non-commercial purposes in accordance with Wiley Terms and Conditions for Self-Archiving.

DOI: https://doi.org/10.1111/jsr.12662

Reference: Aristotelous, Panagiotis, Aphamis, George, Sakkas, Giorgos K., Andreou, Eleni, Pantzaris, Marios, Kyprianou, Theodoros, Hadjigeorgiou, Georgios M., Manconi, Mauro and Giannaki, Christoforos D. (2018) Effects of controlled dehydration on sleep quality and quantity: A polysomnographic study in healthy young adults. Journal of Sleep Research. ISSN 09621105 
Title: Effects of controlled dehydration on sleep quality and quantity: a polysomnographic study in healthy young adults

Authors: Panagiotis Aristotelous ${ }^{1}$, George Aphamis ${ }^{1}$, Giorgos K. Sakkas ${ }^{2}$, Eleni Andreou$^{1}$, Marios Pantzaris ${ }^{3}$, Theodoros Kyprianou ${ }^{1,4}$, Georgios M. Hadjigeorgiou ${ }^{5}$, Mauro Manconi ${ }^{6}$, Christoforos D. Giannaki ${ }^{1,3^{*}}$

Affiliation: ${ }^{1}$ Department of Life and Health Sciences, University of Nicosia, Nicosia, Cyprus; ${ }^{2}$ Faculty of Sport and Health Sciences, University of St Mark \& St John, Plymouth, UK; ${ }^{3}$ The Cyprus Institute of Neurology and Genetics, Nicosia, Cyprus; ${ }^{4}$ General Hospital of Nicosia, Cyprus; ${ }^{5}$ School of Medicine, University of Thessaly, Greece, ${ }^{6}$ Sleep and Epilepsy Center, Neurocenter of Southern Switzerland, Civic Hospital (EOC) of Lugano, Lugano, and Neurology Department Inselspital, Bern University Hospital, Switzerland

*Corresponding Author: ChristoforosD. Giannaki, $\mathrm{PhD}$

$$
\begin{aligned}
& \text { Department of Life and Health Sciences } \\
& \text { University of Nicosia, Nicosia, Cyprus } \\
& 46 \text { Makedonitisas Avenue, CY } 1700 \\
& \text { Tel: +357-22842325; Fax: +357-22842399 } \\
& \text { E-mail: giannaki.c@unic.ac.cy }
\end{aligned}
$$

Word count: 4296

Number of References: 28

Running Head: Dehydration and sleep

Keywords: hydration; sleep; periodic limp movements in sleep; polysomnography

\section{Conflict of interest:None}

Financial disclosure: The study was funded by the European Hydration Institute. EHI Graduate Research Grants 2015-2016. The funding source had no involvement in 
regards to study design, data collection and analysis and interpretation of the data, in the writing of the report as well as in the decision to submit the article for publication. 


\section{Contribution of the authors in the preparation of the manuscript:}

PA: Data collection, Analysis, Interpretation, Drafting and Revising the article, Final Approval

GA: Design, Interpretation, Drafting and Revising the article

GKS: Conception and Design, Analysis \& Interpretation of the data, Drafting and Revising the article, Final Approval

EA: Design, Interpretation, Drafting and Revising the article

MP: Conception and Design, Data analysis, Drafting and Revising the article, Providing intellectual content of critical importance to the work described

TK: Conception and Design, Drafting and Revising the article, Providing intellectual content of critical importance to the work described

GMH: Interpretation, Drafting and Revising the article, Final Approval

MM: Interpretation, Drafting and Revising the article, Providing intellectual content of critical importance to the work described, Final Approval

CDG: Conception and Design, Data collection, Analysis \&Interpretation of the data, Drafting and Revising the article, Final Approval 


\section{Summary}

Dehydration is associated with several alternations in body homeostasis involving both physiological and mental aspects. In addition some studies have reported a negative effect of dehydration on subjectively assessed sleep-related parameters. The aim of the current study was to examine for the first time the effect of controlled dehydration on sleep quality and quantity using the gold standard method of polysomnography. Twelve healthy male volunteers participated in this study (23.4 \pm 0.8 yrs).Participants performed an in house full polysomnographic assessment in two different occasions taking place in random order : a) in a dehydrated state and

b) in a euhydrated state. In the dehydration scenario, the participants were allowed to consume only 1.25 litre of non-caffeinated fluids, while during the euhydrated state participants had to drink at least 3 litre of non-caffeinated fluids during the last 24 hours before the polysomnographic study. Urine specific gravity was assessed by refractrometry on collection day in order to assess hydration status. Participants who did not fulfil the hydration criteria were rescheduled.All participants successfully completed the two polysomnographic studies without any complains or adverse effects reported. No significant differences were found in any of the examined indices of sleep quality and quantity between the dehydration and the euhydration scenario ( $p$ $>0.05)$. This is the first study to show that controlled mild dehydration does not seem to affect sleep quality and quantity in young healthy adults. More research is necessary to further verify these conclusions and whether other parameters are involved in the manifestation of sleep disturbances. 


\section{Introduction}

There is a rapidly growing literature on the negative impact of dehydration on mental and physical processes which is of critical importance for overall health quality and wellbeing. Dehydration is accompanied among other by fatigue, dizziness, cognitive and mood impairment (Masento et al., 2014, Ganio et al., 2011). Surprisingly, around one fifth of the otherwise healthy European adult population seems to be dehydrated (Malisova et al., 2016). On the other hand, poor sleep also negatively affectshealth and quality of life, while chronic sleep restriction and sleep disorders represent a major risk factor for cardiovascular diseases (Sakkas et al., 2015, Shawon et al., 2016).Similarly to dehydration, sleep problems could also affect the general population,-as one in two individuals in the United States and one in three individuals in Europe seem to experience some form of sleeping disorders(Leger et al., 2008).

Quite recently, a considerable attention has been paid to the effect of dehydration on circadian rhythms especially in the sleep-wake cycle (Pross et al., 2014, Pross et al., 2013). Research in this field has documented that reduced water intake could induce excessive daytime sleepiness, fatigue and cognitive dysfunction; all crucial factors for mood, daily living ability, safety and wellbeing. However, we have to acknowledge the fact that the effect of dehydration in some physiological or/and cognitive parameters could be related to the severityof dehydration. For instance, in the study of Szinnai et al. moderate dehydration, induced by water deprivation, did not result in significant impairments in cognitive-motor performance in healthy individuals (Szinnai et al., 2005) while, significant evidence exists regarding the detrimental effect of both Ramadan and alcohol intake on sleep (Leiper et al., 2003, Thakkar et al., 2015).Interestingly, anecdotal reports from alcohol users and people who fast 
during Ramadan, blamed thirst and dehydration as a possible contributor for sleep disturbances. Beside the scarcity of data, a key limitation of this type of data is that sleep has never been assessed by an objective method such as polysomnography (PSG)and therefore it is still not clear whether dehydration could really impact sleep homeostasis. We aimed to approach this crucial question by investigating the effect of controlled dehydration on sleep quality and quantity using the gold standard method of full night polysomnography.

\section{Methods}

\section{Participants}

Twelve healthy young male adults $(23.4 \pm 0.8$ years $)$ participated to the study. Health status was verified by a clinical examination performed by a certified physician. Before the initialization of the project, the first urine sample of the day was collected in order to examine urine specific gravity as an index of the participant's kidneys concentration ability. Exclusion criteria included diagnosed cardiovascular (included hypertension), kidney and sleep-related disorders, obesity (BMI > 30) or any other significant disease known to affect sleep. Anthropometric characteristics were assessed, including weight and height. All volunteers gave their written consent to participate in the study, following a comprehensive explanation of the purpose and the procedures of the study. The study was approved by the National Ethics Committee of Cyprus (No: EЕBK/EП/2016/22).

Study design

Participants underwent two independent full night polysomnographic (PSG) 
assessments at their premises, seven days apart, in random order: oncein a dehydrated state and once in aeuhydrated state. The participants were instructed to record and follow the same dietary intake for the last $24 \mathrm{~h}$ replicating thus the first visit day.Fluid and water intake were controlled as appropriate and according to the respective scenario.

In the dehydration scenario, on testing day, the participants were given fluids (mineral water) on specific intervals (total amount of fluids $5 \times 250 \mathrm{ml}=1.25$ litre) during the last 24 hours before sleep study, in order to induce controlled mild dehydration (Figure 1). In the euhydration scenario, mineral water consumption was standardized (500 $\mathrm{ml}$ at meal time points and $250 \mathrm{ml}$ at snack and hydration time points) to achieve a total daily intake of 3 liters during the last 24 hours before the sleep study and according to international guidelines (Institute of Medicine of the National Academies, 2005)(Figure 1).In addition, an individualized diet (including liquids) plan was prepared by a qualified clinical dietitian-nutritionist and was followed exactly under both testing conditions in order to eliminate potential effects of varying diet on sleep. The participants were free to eat food of their choice, avoiding a list of food high in water content or salt. Overall, the participants were not allowed to consume high-water-containing foods, alcohol and caffeine. A urine sample, both before bedtime and at awakening, was obtained in order to verify the hydration status of the participants in both scenarios.

From19.30h of the day before the PSG study, until the next day at $7.00 \mathrm{~h}$ fluid and food restriction was applied to all the participants (last meal-low water containing was consumed at 19:30h). Thereafter, the participants were allowed to drink ad libitum. 


\section{Polysomnographic study}

The polysomnographic (PSG) study was performed according to the guidelines of theAmerican Academy of Sleep Medicine(Berry et al., 2015)at the participants preferred premises in order to keep as constant as possible their sleep routine and environment. Lights "off" was established at22.00h and lights "on" at 07.00h, and polysomnograms (Somnoscreen, Somnomedics GmbH, Randersacker, Germany)were collected overnight. The PSG recording included the following parameters: electroencephalogram(according to the international 10-20 system: F3, F4, C3, C4, $\mathrm{P} 3, \mathrm{P} 4, \mathrm{O} 1, \mathrm{O} 2$, ground (at AFz) and a reference electrode at position $\mathrm{FCz}$ ); right and left electrooculogram; submental and both tibialis anterior muscles; body position; electrocardiogram; thoracic and abdominal efforts (piezoelectric transducers); oronasal airflow (thermistor); and oxygen saturation. Due to the domestic environment, no video and sound were assessed. All PSG-related outcome measures including sleep stages were determined using the standard scoring criteria(Berry et al., 2015).

Heart rate related parameters during sleep was recorded via an electrocardiogram (ECG)-part of the PSG device. Minimum, average and maximum HR were recorded. Polysomnographic scoringand offline analyses were performed blinded in respect to the scenario of the study.

Hydration status assessment: urine specific gravity measurement

Urine specific gravity was assessed by a handheld refractrometer (Medline Scientific, Oxfordshire, UK) on collection day (before and after the PSG study) (Figure 1). 
Dehydration cut-off value was set at 1.020 for specific gravity(American College of Sports et al., 2007). Participants who did not fulfil the hydration criteria were rescheduled.

Water intake assessment

Total water intake coming from fluids and solid food was later estimated based on the $24 \mathrm{~h}$ food dietary records provided, using Dietplan.6 software by a qualified clinical dietitian-nutritionist.

\section{Statistical analysis}

Comparisons between euhydrated vs. dehydrated data were made using paired t-test. Pearson correlation test was used to assess the relationship between SG values preand post the PSG study and PSG-derived indices. All the statistical analyses were performed using the SPSS statistical software for windows version 19.All data are reported as mean \pm standard deviation. The level for statistical significance was set atp $=0.05$.

\section{Results}

Table 1 shows the main features of the participants. All participants successfully performed the two polysomnographic studies without any adverse effects or complaints being reported. 
Urine specific gravity as assessed before the start of the PSG study, as well as the one assessed in the morning after the PSG study was significantly higher in the dehydration scenario compared with the euhydration scenario (before PSG: $1.028 \pm$ 3.1 vs $1.014 \pm 4.4 ; \mathrm{p}=0.000$; after PSG: $1.033 \pm 4.5$ vs $1.014 \pm 3.3 ; \mathrm{p}=0.000$ ). The mean ambient temperature and humidity in the dehydration scenario did not significantly differ from the respective temperature in the euhydration scenario ( $\mathrm{T}$ at 10 pm: $23.5 \pm 3.6$ vs $22.8 \pm 3.3 ; \mathrm{p}=0.277$; humidity at $10 \mathrm{pm}: 51.8 \pm 7.1$ vs $55.2 \pm$ $8.7, \mathrm{p}=0.137)$.

According to the $24 \mathrm{~h}$ food and fluid record analysis, water intake averaged between $3.437 \pm 0.169 \mathrm{ml}$ and $1362.9 \pm 228.0$ mlfor the euhydration and dehydration scenario respectively.

Table 2 shows the PSG sleep parameters. No significant differences were found in any of the examined variables between the two scenarios. The respiratory and heart rate data are presented in Table 3. Again, no significant differences were found in any of the examined variables between the two scenarios. Finally, the data regarding leg movements are shown in Table 4. Again, no significant differences were found in any of the examined variables between the two scenarios. Finally, no significant correlations were found between dehydration status (assessed by SG) and PSGderived indices. 


\section{Discussion}

According to our knowledge, this is the first controlled sleep study evaluating the effect of mild dehydration on objectively assessed sleep related parameters using the polysomnographic gold standard approach. It has been found that a single controlled mild dehydration did not impose any changing effect on sleep related parameters in a sample of healthy young adults, shedding thus more light in some recent contradicting published results. It is possible that the current evidence could have been affected by the nature of this preliminary study design as a larger sample size, and a more severe level of dehydration could have affected the present outcomes.

It is important to note that all previous studies have used subjective tools such as questionnaires and sleep diaries in order to assess sleep quality in the various dehydration/water intake studies. Pross et al., by increasing water intake into habitual low drinkers (healthy young adults) observed an improvement on mood, sleepiness, fatigue, thirstiness and confusion(Pross et al., 2014). On the other hand, reducing water intake of habitual high drinkers resulted in reductions on contentedness, calmness, positive emotions and vigour activity (Pross et al., 2014). In another study, conducted on 20 female healthy subjects, the same authors showed that 24 hours of mild fluid deprivation increased sleepiness, fatigue and confusion and decreased alertness(Pross et al., 2013).

Previous studies have tried to connect Ramadan with self-reported sleep disturbances(Leiper et al., 2003). Roky and colleagues, documented a significant impact of Ramadan intermitted fasting on polysomnographic sleep parameters of healthy young adults (Roky et al., 2001). In particular, total sleep time was 
significantly decreased, proportion and structure of non-REM sleep was also altered and finally REM sleep duration significantly decreased. Ramadan requires, inter alia, restriction of water from sunrise to sunset, and therefore dehydration could be resulted despite the fact that the participants drink a lot of fluids after sunset minimizing the negative water balance (Leiper et al., 2003).

Kempton et al. elicited $2.2 \%$ body weight dehydration via exercise (Kempton et al., 2009). Their findings suggest that abnormal regulation of fluid intake may adversely affect brain structure and function. In the present study, fluid intake was reduced to 1.25 L over the 24 hour preceding the main trial, which is half of the European (EFSA, 2010) and almost one third of the US (Institute of Medicine of the National Academies, 2005) recommended water intake for males. As described in the methods section, in the present study, dehydration was monitored via USG measurements. The reduced water intake in the present study did induce dehydration as USG was 1.028. A USG between 1.021 and 1.030 corresponds with a level of dehydration of $3 \%$ to $5 \%$ of body mass (Casa et al., 2000). In the present study, USG was 1.028, indicating a small but significant degree of dehydration that according to previous studies (Kempton et al., 2009) may potentially elicit changes in brain structure and function. Therefore it seems that the degree of dehydration was not the pivotal player in the lack of significant changes between the two scenarios.

However, a notable but not statistically significant observation of the current study is that the percentage of REM decreased of approximately $8 \%$ and the percentage of light sleep increasedby7\%, when the participants were dehydrated. In future studies, with a larger cohort, and/or with female subjects, these findings may become 
significant and may provide insights into why dehydrated individuals(especially elder people and patients) often report feelings of exhaustion, and cognitive and mood impairment(Masento et al., 2014). Finally, potential thirstiness feeling experienced by the participants during the dehydration state seems to not affect the amount of time it took the participants to fall asleep.

The current study has shown that there is no statistical association between dehydration status and respiratory indices during sleep even though, an approximately twofold increase in desaturation events occurred in the dehydration scenario compared with the euhydration one highlighting thus the clinical significance of this phenomenon in patients with respiratory disorders. In dehydration, the blood viscosity and hematocrit values increase due to fluid loss. In a polysomnographic study in patients with obstructive sleep apnea, high hematocrit levels were significantly correlated with low oxygen saturation (Choi et al., 2006). Moreover, OSAS induce dehydration with xerostomia as one of the prominent clinical feature.

Periodic limb movements in sleep (PLMS)are repetitive, stereotypical and not volitional leg jerks occurring during sleep and detected by PSG. PLMS are considered a supportive criterion for the diagnosis of Restless Legs Syndrome-RLS (referred also as Willis-Ekbom disease)which is common in the general population (idiopathic RLS), and even more frequent in many other conditions such as pregnancy, iron deficiency, chronic renal disease, multiple Sclerosis, hypertension, diabetes and Parkinson disease (secondary RLS) (Giannaki et al., 2014, Trenkwalder et al., 2016). PLMS is present in over $80 \%$ of patients with RLS (Allen et al., 2003). Despite nonsignificant differences, both the PLMS index and number increased in the dehydration 
scenario. As PLMS is associated, among others, with the development of aggravation of existing cardiovascular diseases (Giannaki et al., 2013), more research with larger sample size is required in order to uncover the potential impact of dehydration on leg movements during sleep.

The outcomes of the current study reveal that moderate dehydration did not significantly affect heart rate, confirming previous data in healthy young adults (Schwabe et al., 2007). This could be explained by the gender effect which may exist regarding the response of sympathetic nervous system (SNS) in dehydration conditions, as a negative effect of dehydration was reported only in women and not to men (Schwabe et al., 2007).In addition, inter-individual variability in regards to SNS control of heart rate may explain the failure of dehydration to induce changes in heart rate in some subjects (Charkoudian and Rabbitts, 2009).

Alternatively, the absence of a significant change in heart rate may have been associated with the homeostatic mechanisms of the human body during sleep versus wakefulness - in particular, those related to changes in body position between the two states. In the study of Gonzalez-Alonso et al., dehydration induced significant changes in heart rate and blood pressure during upright exercise, whilst no significant changes were observed during supine exercise (Gonzalez-Alonso et al., 1999).Therefore, the potential negative effects of dehydration on physiological parameters that are known to occur during awakeness could be masked under sleeping conditions or/and body position in healthy individuals. 
Both, water intake derived by the food record and specific gravity values ensure as much as possible the hydration status of the participants in the two scenarios. The mean specific gravity value obtained before the start of the PSG examination was 1.028 and can be classified as slight dehydration (Casa et al., 2000).It would be interesting to investigate in future studies the effect of severe dehydration on sleep parameters or whether dehydration due to extreme sweating would have a different impact on aspects related to sleep and sleepiness.

In the current study some strengths and weaknesses need to be mentioned. This is the first study using the gold standard methodology of assessing the impact of mild selfinduced dehydration on parameters relates to sleep. In addition, dehydration and euhydration status were carefully controlled by standard procedures and therefore the intra participants' differences were minimized. Despite the fact that PSG is considered to be the gold standard method to measure sleep objectively, we should note the possibility that in-home PSG could be more susceptible to artifact (and therefore less sensitive to any type of manipulation) in comparison to standard in-laboratory PSG. Unfortunately, the current study's design did not include the assessment of mood and subjective feelings. Therefore, we lost the opportunity to assess how the participants felt during the dehydration night. Moreover, we did not assess whether cognitive performance was altered upon waking in the dehydration scenario. Evidence exists regarding a negative effect of even mild level of dehydration to cognitive performance(Ganio et al., 2011, Wilson and Morley, 2003), however, we should note that other studies did not confirm such hypothesis(Szinnai et al., 2005).In addition we did not perform a third PSG study under "the usual" hydration status of the participants to address potential intra-individual differences. Unfortunately due to 
technical difficulties, total body water and subsequently intracellular and extracellular water content were not assessed to further verify hydration status. Despite the negative effect of dehydration observed in the current study, we should note the participants were healthy young adults (males). It would be interesting to examine the effect of dehydration on other group of the population such as elderly individuals and patients with chronic diseases including patients with sleep disorders. In addition, since studies in healthy young women have revealed that even moderate dehydration could negatively affect various mental and physical parameters(Armstrong et al., 2012)it would be interesting to replicate the present study with female subjects.

In conclusion, mild dehydration did not induce any significant alternations in polysomnographic indices of sleep quality and quantity in healthy young adults as it was assessed by a full night Polysomnography. More research is needed before a safe conclusion can be drawn in regards to the association between dehydration and sleep.

Acknowledgments: We would like to thank all the participants who volunteered for the purposes of the study as well as Ms. Maria Kotsoglanidis and Ms. Evangelia Hadjicharalambous for their help and support. 


\section{References}

Allen, R. P., Picchietti, D., Hening, W. A.et al. Restless legs syndrome: diagnostic criteria, special considerations, and epidemiology. A report from the restless legs syndrome diagnosis and epidemiology workshop at the National Institutes of Health. Sleep Med, 2003, 4: 101-119.

American College of Sports, M., Sawka, M. N., Burke, L. M.et al. American College of Sports Medicine position stand. Exercise and fluid replacement. Med Sci Sports Exerc, 2007, 39: 377-390.

Armstrong, L. E., Ganio, M. S., Casa, D. J.et al. Mild dehydration affects mood in healthy young women. $J$ Nutr, 2012, 142: 382-388.

Berry, R. B., Brooks, R., Gamaldo, C. E.et al. The AASM Manual for the Scoring of Sleep and Associated Events: Rules, Terminology and Technical Specifications, Version 2.1. Darien, Illinois: American Academy of Sleep Medicine, 2015

Casa, D. J., Armstrong, L. E., Hillman, S. K.et al. National athletic trainers' association position statement: fluid replacement for athletes. Journal of athletic training, 2000, 35: 212-224.

Charkoudian, N. and Rabbitts, J. A. Sympathetic neural mechanisms in human cardiovascular health and disease. Mayo Clin Proc, 2009, 84: 822-830.

Choi, J. B., Loredo, J. S., Norman, D.et al. Does obstructive sleep apnea increase hematocrit? Sleep Breath, 2006, 10: 155-160.

Ganio, M. S., Armstrong, L. E., Casa, D. J.et al. Mild dehydration impairs cognitive performance and mood of men. Br J Nutr, 2011, 106: 1535-1543.

Giannaki, C. D., Hadjigeorgiou, G. M., Karatzaferi, C., Pantzaris, M. C., Stefanidis, I. and Sakkas, G. K. Epidemiology, impact, and treatment options of restless legs syndrome in end-stage renal disease patients: an evidence-based review. Kidney Int, 2014, 85: 1275-1282.

Giannaki, C. D., Karatzaferi, C., Hadjigeorgiou, G. M., George, K. P., Stefanidis, I. and Sakkas, G. K. Periodic limb movements in sleep and cardiovascular disease: time to act. Front Neurol, 2013, 4: 97.

Gonzalez-Alonso, J., Mora-Rodriguez, R. and Coyle, E. F. Supine exercise restores arterial blood pressure and skin blood flow despite dehydration and hyperthermia. Am J Physiol, 1999, 277: H576-583.

Kempton, M. J., Ettinger, U., Schmechtig, A.et al. Effects of acute dehydration on brain morphology in healthy humans. Human brain mapping, 2009, 30: 291298.

Leger, D., Poursain, B., Neubauer, D. and Uchiyama, M. An international survey of sleeping problems in the general population. Current medical research and opinion, 2008, 24: 307-317.

Leiper, J. B., Molla, A. M. and Molla, A. M. Effects on health of fluid restriction during fasting in Ramadan. Eur J Clin Nutr, 2003, 57 Suppl 2: S30-38.

Malisova, O., Athanasatou, A., Pepa, A.et al.Water Intake and Hydration Indices in Healthy European Adults: The European Hydration Research Study (EHRS). Nutrients, 2016, 8: 204.

Masento, N. A., Golightly, M., Field, D. T., Butler, L. T. and Van Reekum, C. M. Effects of hydration status on cognitive performance and mood. Br J Nutr, 2014, 111: 1841-1852. 
Institute of Medicine of the National Academies. Dietary reference intakes for water, potassium, sodium, chloride, and sulfate. Washington DC: The National Academies Press. 2005. www.nap.edu.

Pross, N., Demazieres, A., Girard, N.et al. Effects of changes in water intake on mood of high and low drinkers. PLoS One, 2014, 9: e94754.

Pross, N., Demazieres, A., Girard, N.et al. Influence of progressive fluid restriction on mood and physiological markers of dehydration in women. Br J Nutr, 2013, 109: 313-321.

Roky, R., Chapotot, F., Hakkou, F., Benchekroun, M. T. and Buguet, A. Sleep during Ramadan intermittent fasting. J Sleep Res, 2001, 10: 319-327.

Sakkas, G. K., Giannaki, C. D., Karatzaferi, C.et al. Current trends in the management of uremic restless legs syndrome: a systematic review on aspects related to quality of life, cardiovascular mortality and survival. Sleep Med Rev, 2015, 21: 39-49.

Schwabe, L., Szinnai, G., Keller, U. and Schachinger, H. Dehydration does not influence cardiovascular reactivity to behavioural stress in young healthy humans. Clin Physiol Funct Imaging, 2007, 27: 291-297.

Shawon, M. S., Perret, J. L., Senaratna, C. V., Lodge, C., Hamilton, G. S. and Dharmage, S. C. Current evidence on prevalence and clinical outcomes of comorbid obstructive sleep apnea and chronic obstructive pulmonary disease: A systematic review. Sleep Med Rev, 2016

Szinnai, G., Schachinger, H., Arnaud, M. J., Linder, L. and Keller, U. Effect of water deprivation on cognitive-motor performance in healthy men and women. Am J Physiol Regul Integr Comp Physiol, 2005, 289: R275-280.

Thakkar, M. M., Sharma, R. and Sahota, P. Alcohol disrupts sleep homeostasis. Alcohol, 2015, 49: 299-310.

Trenkwalder, C., Allen, R., Hogl, B., Paulus, W. and Winkelmann, J. Restless legs syndrome associated with major diseases: A systematic review and new concept. Neurology, 2016, 86: 1336-1343.

Wilson, M. M. and Morley, J. E. Impaired cognitive function and mental performance in mild dehydration. Eur J Clin Nutr, 2003, 57 Suppl 2: S24-29. 


\section{Tables and Figures}

Figure 1: Study design

Abbreviations: AL, ad libitum water intake; SG, specific gravity assessment; PSG, polysomnographic study 
Table 1: General characteristics of the participants

\begin{tabular}{lc}
\hline \hline Variables \\
\hline $\mathrm{N}$ & 12 \\
\hline Age $(\mathrm{yr})$ & $23.4 \pm 0.8$ \\
\hline Body mass index $\left(\mathrm{Kg} / \mathrm{m}^{2}\right)$ & $25.5 \pm 2.5$ \\
\hline \hline
\end{tabular}

All data are mean \pm SD. 
Table 2: Sleep quality and quantity indicesdivided into euhydration and dehydration scenario

\begin{tabular}{lccc}
\hline \hline Variables & $\begin{array}{c}\text { Euhydration } \\
\text { Scenario }\end{array}$ & $\begin{array}{c}\text { Dehydration } \\
\text { Scenario }\end{array}$ & p values \\
\hline Sleep duration (min) & $388.3 \pm 54.6$ & $379.6 \pm 70.7$ & 0.635 \\
\hline Sleep efficiency (\%) & $85.9 \pm 4.4$ & $84.5 \pm 5.7$ & 0.294 \\
\hline Light sleep (\%) & $41.2 \pm 15.5$ & $48.0 \pm 11.9$ & 0.089 \\
\hline Deep sleep (\%) & $23.6 \pm 9.5$ & $23.8 \pm 7.4$ & 0.938 \\
\hline Stage 1 (\%) & $19.8 \pm 12.0$ & $22.7 \pm 9.8$ & 0.363 \\
\hline Stage 2 (\%) & $21.3 \pm 11.0$ & $25.2 \pm 7.2$ & 0.218 \\
\hline Stage 3 (\%) & $23.6 \pm 9.5$ & $23.7 \pm 7.5$ & 0.961 \\
\hline REM Sleep (\%) & $35.1 \pm 18.8$ & $28.1 \pm 9.7$ & 0.119 \\
\hline Sleep latency (min) & $21.5 \pm 22.7$ & $21.8 \pm 20.6$ & 0.946 \\
\hline Arousals index & $10.0 \pm 3.6$ & $10.5 \pm 3.3$ & 0.455 \\
\hline \hline
\end{tabular}

All data are mean \pm SD. Abbreviations: REM, rapid eye movements 
Table 3: Polysomnography derived-respiratory indices and heart rate indices divided into euhydration and dehydration scenario

\begin{tabular}{lccc}
\hline Variables & $\begin{array}{c}\text { Euhydration } \\
\text { Scenario }\end{array}$ & $\begin{array}{c}\text { Dehydration } \\
\text { Scenario }\end{array}$ & p values \\
\hline OSA index & $3.3 \pm 5.9$ & $3.1 \pm 4.0$ & 0.741 \\
\hline Central Apnea index & $0.0 \pm 0.0$ & $1 \pm 2.2$ & 0.154 \\
\hline Mixed Apnea Index & $0.02 \pm 0.08$ & $0.06 \pm 0.14$ & 0.096 \\
\hline AHI & $3.0 \pm 6.2$ & $3.6 \pm 4.7$ & 0.425 \\
\hline RDI & $3.1 \pm 6.1$ & $3.7 \pm 4.6$ & 0.365 \\
\hline Desaturations number & $7.2 \pm 14.7$ & $16.9 \pm 40.9$ & 0.233 \\
\hline Minimal SpO 2 & $86.7 \pm 6.1$ & $88.1 \pm 6.5$ & 0.479 \\
\hline Maximum heart rate & $93 \pm 10$ & $92 \pm 10$ & 0.507 \\
\hline Minimum heart rate & $41 \pm 8$ & $40 \pm 4$ & 0.214 \\
\hline Average heart rate & $51 \pm 8$ & $50 \pm 6$ & 0.148 \\
\hline \hline
\end{tabular}

All data are mean \pm SD. Abbreviations: OSA, obstructive sleep apnea; AHI, apneahypopnea index; RDI, respiratory disturbance index; $\mathrm{SpO}_{2}$, blood oxygen saturation 
Table 4: Leg movement indices

\begin{tabular}{lccc}
\hline \hline Variables & $\begin{array}{c}\text { Euhydration } \\
\text { Scenario }\end{array}$ & $\begin{array}{c}\text { Dehydration } \\
\text { Scenario }\end{array}$ & p values \\
\hline Total leg movements & $115.8 \pm 71.1$ & $131.2 \pm 48.2$ & 0.239 \\
\hline Total leg movements index & $17.6 \pm 10.3$ & $20.2 \pm 7.2$ & 0.143 \\
\hline PLMS & $54.3 \pm 44.1$ & $61.2 \pm 31.7$ & 0.483 \\
\hline PLMS index & $8.0 \pm 6.3$ & $9.4 \pm 4.6$ & 0.296 \\
\hline PLMS with arousals & $4.9 \pm 4.3$ & $5.2 \pm 2.2$ & 0.839 \\
\hline PLMS with arousals index & $0.6 \pm 0.7$ & $0.8 \pm 0.3$ & 0.433 \\
\hline Leg movements with arousals & $7.4 \pm 6.5$ & $10.1 \pm 5.1$ & 0.092 \\
\hline $\begin{array}{l}\text { Leg movements with arousals } \\
\text { index }\end{array}$ & $1.2 \pm 1.0$ & $1.5 \pm 0.7$ & 0.142 \\
\hline \hline
\end{tabular}

All data are mean \pm SD. Abbreviations: PLMS, periodic limb movements in sleep 